Case Report

\title{
Onychomadesis (Nails Shedding) Secondary to Enterovirus Herpangina Like Infection and Without the Skin Manifestations of Hand Foot Mouth Disease
}

\author{
Sam Hassan \\ Department of Paediatrics, Mediclinic City Hospital, Dubai Health Care City, Dubai \\ Email address: \\ sam.hassan@mediclinic.ae
}

To cite this article:

Sam Hassan. Onychomadesis (Nails Shedding) Secondary to Enterovirus Herpangina Like Infection and Without the Skin Manifestations of Hand Foot Mouth Disease. American Journal of Pediatrics. Vol. 5, No. 3, 2019, pp. 78-81. doi: 10.11648/j.ajp.20190503.11

Received: May 6, 2019; Accepted: June 10, 2019; Published: June 26, 2019

\begin{abstract}
Onychomadesis or onycholysis is a newly recognized complication in the course of viral infections particularly the Hand Foot Mouth Disease (HFMD) in children and adults. Onychomadesis is characterized by proximal separation of the nail plate from the nail matrix due to temporary cessation of growth of the nail matrix. The etiology of onychomadesis is including infections, trauma, medications side effects, systemic diseases and idiopathic. The association of the HFMD and the onychomadesis first reported in the year 2000. Since then, few studies and case reports published illustrating the relationship of the HFMD and the resultant onychomadesis 4 to 8 weeks after the resolution of the HFMD. HFMD is a relatively common viral infection, especially in children of preschool age. It is usually presented with characteristic eruption on hands, feet and mouth. Out breaks may happen in nurseries and schools. HFMD usually caused by enteroviruses, most commonly, Coxsackie virus A (CVA) and enterovirus 71 (EV71). It is usually self-limiting disease however; serious neurological complications have been reported with EV71. The enteroviruses that cause HFMD and nail changes may cause herpangina which is unlike the HFMD, usually characterized by mouth spots on the soft palate without the skin manifestations, however onychomadesis was not reported before with herpangina. This case presented with no HFMD eruption but small red spots on the soft palate consistent with herpangina. Enterovirus was isolated from throat swab and the onychomadesis occurred 1 month after the resolution of the soft palate spots. It is the first case report about nail changes occurring after herpangina presentation. It is therefore important that in patients presented with onychomadesis to consider the review of the patient's history not only for HFMD but herpangina and possible other EV infections, 8 weeks before the nail changes, to avoid unnecessary referrals, concerns and over investigations. The exact mechanism of the nail changes is not yet known and a review of theories behind the nail damage will be considered in this report.
\end{abstract}

Keywords: Hand Foot Mouth Disease, Herpangina, Enetrovirus Infection, Nails, Onychomadesis

\section{Introduction}

Onychomadesis is characterized by separation of the nail plate from the matrix due to a temporary arrest in the nail matrix activity. Interruption of the nail matrix function may result from repetitive trauma, exposure to chemicals such as household cleaners, fungal infections, herpes simplex infection, reaction to medications such as chemotherapy, tetracycline, fluoroquinolone, and chlorpromazine, systemic diseases such as thyroid diseases, amyloidosis, diabetes mellitus, erythropoietic porphyria, hyperhidrosis, pellagra,
Reiter's syndrome, leprosy, sarcoidosis, scleroderma, yellow nails syndrome, iron and vitamin deficiencies, skin conditions such as psoriasis and can be idiopathic or congenital. Such long list of causes can lead to over concerns, over investigations and unnecessary referrals. However, such concerns can be eliminated by careful history of viral infections such as EV some 8 weeks preceded the nail changes. Not long time ago, we came to know about the association of onychomadesis and HFMD in the year 2000 [1]. Following 
that few cases and articles were reported about the association of the typical skin eruption of HFMD and the onychomadesis that presented at least 4 weeks later [2, 3 and 4]. HFMD usually spreads through close personal contact, airborne particles (coughing and sneezing), and contact with contaminated objects and surfaces. Important to know that infection can be transmitted by contaminated swimming pool [5]. The viruses that cause HFMD can be found in an infected person's nose or throat secretions, blister fluid and stool. It may cause widespread outbreaks in nurseries and schools. Some individuals remained asymptomatic yet they can spread the infections to others. HFMD may lead to onychomadesis few weeks after the disease subsided, however, it is not known if the career and asymptomatic cases of HFMD may contribute to the causation of the idiopathic type of onychomadesis. Herpangina is another viral infection caused by the same genus of EV which mainly presented with fever and red spots on soft palate that might ulcerate and become painful. Both HMFD and herpnagina occur in children and may cause outbreaks in nurseries and schools.

This case report is the first report of herpangina infection by EV that presented without the typical skin eruption of HFMD and later on caused onychomadesis about 4 weeks when the herpangina spots subsided. Both HFMD and herpangina are common acute febrile viral infection that presents as a vesicular eruption in mouth, however the typical HFMD manifest with hands, feet, buttocks, and/or genitalia eruptions. Oral eruption is second commonest to palms and soles vesicles in HFMD. Fever is not always reported and it may range from $38.5^{\circ} \mathrm{C}$ to $41.0^{\circ} \mathrm{C}$. Interestingly patients with no fever is likely to have more affected nails than patients with fever. [6].

Coxsackievirus A type 16 (CVA16) is the commonest etiologic agent involved in most cases of HFMD, but the illness is also associated with coxsackievirus A5, A7, A9, A10, B2, and B5 strains. Enterovirus 71 (EV-71) has caused outbreaks of HFMD that may be rarely associated with neurologic involvement such as polio-like syndrome, aseptic meningitis, encephalitis, encephalomyelitis, acute cerebellar ataxia, acute transverse myelitis, Guillain-Barré syndrome, opsomyoclonus syndrome, benign intracranial hypertension. The diagnosis of HFMD is clinical however virus may be isolated by Polymerase Chain Reaction (PCR) from samples from oral mucus, stool, skin lesions, and rectal swab. Enterovirus infection may present with upper respiratory tract and/or gastrointestinal infections alone. This case was presented with painful erythematous spots in the soft palate without skin eruptions about 6 weeks before the patient presented with onychomadesis involving all fingers nails in both hands. The nail changes initially presented as a transverse line at the base of the nails with elevation of the nail bed followed by shedding of the whole nails and new nail emerging after 1 month. The presentation of this case was more similar to herpangina than to the typical HFMD. Both HFMD and herpangina caused by enteroviral infection. The genus Enterovirus belongs to the Picornaviridae family. Within the genus, there are 12 species, which contain the coxsackievirus, echovirus, human rhinovirus, and poliovirus serotypes. The main strains which cause HFMD are coxsackievirus A16 and enterovirus A71. Coxsackievirus A1-6, 8, 10, and 22 are frequently associated with herpangina [7]. Echoviruses have also been implicated in both syndromes. Enteroviruses are small, non-enveloped, single positive-strand RNA viruses. They are capable of surviving in a wide $\mathrm{pH}$ range and retain infectivity in temperatures up to $50^{\circ} \mathrm{C}$. These characteristics make them capable of surviving in the environment for relatively extended periods. Both herpangina and HFMD are highly contagious infections, usually self-limiting but rarely may cause serious cardiac and neurological complications [8]. Humans are the only natural host of these viruses [9]. It is therefore both HFMD and herpangina need to be sought in the history of patients presented with onychomadesis.

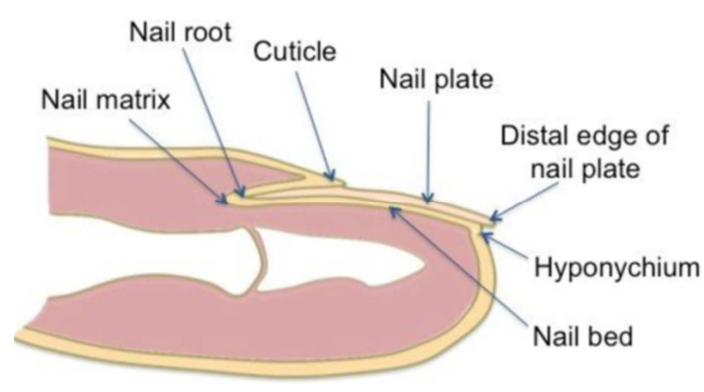

Figure 1. Normal nail structures.

The exact mechanism of nail changes following HFMD viral infections is not well known. Normally the nails formed constantly from the Matrix at the base of the nail. This part represents the growth plate which lay the nail as it grows (figure 1). Interestingly that the Matrix is immune-locally protected from germs and infections by a layer of skin called the Cuticle, however, the exact mechanism of such protection is not very well known. Viral infection may play a role in breaching this local immunological protection. It is therefore, Enterovirus infection may interrupt the nail matrix activity which is a continuous process, leading to separation of the matrix from the nail bed and nail plate. The exact cause of interruption is not very well known and whether it is a direct effect of the virus or due to post viral immunological mechanism is yet to be determined. The whole process of the finger nails growth takes from 1 to 6 months to grow back to full nail after falling. In this case it is possible that the virus produced direct effect (lesions) on the nail matrix similar to the soft palate. This case that presented with herpangina spots on soft palate without the skin eruption of HFMD and caused by $\mathrm{EV}$ was complicated by nail changes similar to that happening in the typical cases of HFMD. It seems such presentation might have been under reported. It is therefore advised that clinicians and health professionals assessing a child with nail oncholysis should take a careful history about viral infections such as herpangina and URTI few weeks before and not only about HFMD. On the other hand parents should be advised about nail changes weeks after EV and CAV infections including HFMD and herpangina to avoid 
unnecessary stress and over investigations or referrals.

\section{Case Report}

A previously healthy 3 years and 10 months old boy presented with a history of nail changes for 2 weeks involving all fingers to a variable degree which are not painful. It started as transverse rims at the base of the nail plate with progressive elevation of the nail bed before it starts to shed from the proximal matrix and replaced by a new nail later on. Initially the damage started as a little ridge in one thumb nail in which the parents thought it was due to unnoticed trauma. One week later all other nails started to get similar changes. After 1 month all nails shed fully and replaced with new thinner healthy nails. There were no similar changes in the feet nails. The patient had no history of systemic illness, no exposure to chemicals, trauma, or medication. There were no significant past history and the child was healthy with normal growth and development for age with up to date immunization. On presentations the vital signs were normal with a temperature of $37.0^{\circ} \mathrm{C}$, pulse rate 70 per minute, blood pressure $96 / 60$, $\mathrm{SaO} 2$ in air $99 \%$, respiratory rate 20 per minutes. Systemic examinations including neurological, skin mucus membranes were normal accept the nail changes. All hand nails were affected but not the feet. There were transverse ridges similar to the Beau's lines at the base of each nail especially the big one and some of them started to shed proximally (figure 2). Six weeks later new nails formed and the old damaged ones shed completely (figure 3). One month before the nail changes, the patient presented to the Emergency Department with fever, rhinorrhea, abdominal pain, reduced oral intake, one episode of vomiting and mildly concentrated urine. There was no family history of infection but he attended nursery where some cases of upper respiratory tract infections reported. At that time his vital signs including temperature were normal, urine dipstick was normal and he was discharged home with paracetamol syrup if needed. Two days later the patient attended the Out-Patient Department with oral pain on feeding and found to have small red spots in the soft palate consistent with herpangina and thought to be related to a viral cause. His temperature was $36.6^{\circ} \mathrm{C}$ and the rest of vital signs were normal. The systemic examination was normal and no skin rash apart from the red spots in the soft palate consistent with herpnagina presentation. A urine test and culture were normal. A respiratory polymerase chain reaction swab from the nose was done which was positive for Enterovirus. Streptococcal rapid antigen swab from throat was negative. The patient was given Ibuprofen and Paracetamol syrup and topical oral Medijel (Lidocaine hydrochloride) for the sore throat. On follow up the patient recovered fully and remained healthy and active. He got a younger brother 10 months old who is healthy apart from small hemangioma on the face that is treated with propranolol. Both parents are healthy and there is no family history of significance.

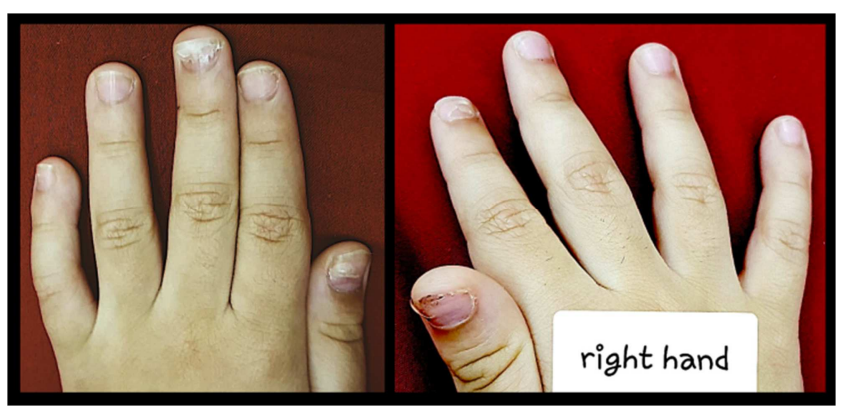

Figure 2. Onychomadesis after 4 weeks from EV URTI.

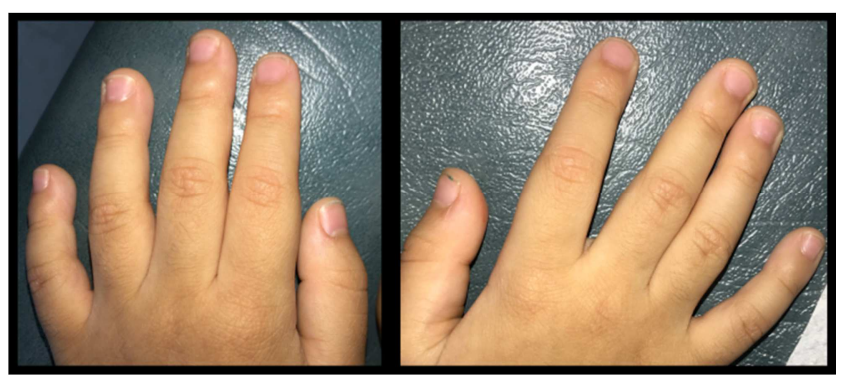

Figure 3. new nails formation.

\section{Discussion}

The first reports of the association of HFMD and the onychomadesis was reported in the year 2000. Many cases reported thereafter, presented with complete or partial presentation of HFMD few weeks before the oncholysis started [10-11]. This case report is different that the oncholysis happened after enterovirus herpangina like infection and upper respiratory tract infection but no skin manifestation of HFMD. It is therefore, showed that oncholysis can happen from the same viruses that can cause HFMD when presented as herpangina and URTI without the skin manifestations of HFMD. The HFMD is caused by a group of enteroviruses most commonly Coxsackie Virus A (CVA) and Enterovirus EV71 [12]. It is usually a mild febrile illness of infants and young children and self-limiting in 7 to 10 days. The typical presentation includes vesicular eruption on palms, soles, buttock and painful ulceration in the oral mucosa. In some cases HFMD may rarely cause sever neurological complications such as meningitis, encephalitis, encephalomyelitis, neurogenic pulmonary edema, and circulatory failure. Enterovirus infection especially EV71 may be associated with severe, potentially life-threatening complications in children, such as brainstem encephalitis, aseptic meningitis, encephalomyelitis, flaccid paralysis, heart failure, and respiratory failure [13].

Nail changes associated with febrile infections was first described in 1846 by Beau [14]. Beau's lines are transverse ridges that can happen in many febrile infections and particularly with HFMD and Kawasaki disease in children. The exact mechanism of the onychomadesis after febrile illness is not well known. However it is probably related to post inflammatory periungual localized reaction or direct viral infection on nail matrix leading to temporary cessation of the nail matrix growth (figure 1). Complete and temporary 
inhibition of nail growth at least for 1 to 2 weeks, leads to separation of the nail bed from the nail matrix and resultant Beau's lines then gradual nail shedding. New nail then formed following resumption of the nail matrix function. Nail shedding due to HFMD occur without scaring unlike tumors, erosive lichen planus and sever systemic diseases. If onychomadesis occurs recurrently other systemic causes should be investigated [15].

Onychomadesis was not reported before with herpangina or URTI. This case illustrates that EV infections other than typical HFMD such as herpangina or URTI may lead to oncholysis in a similar way as the typical HFMD. More studies are required to determine the serotypes of the viruses and the pathogenesis that can cause onychomadesis.

\section{Conclusion}

Clinicians should be aware that EV infections other than HFMD such as herpangina and URTI may later cause nail damages similar to the one caused by the typical HFMD. It is therefore important to consider these infections in the differential diagnosis of children presented with onychomadesis. On the other hand, advising the parents or patients about possible nail changes few weeks later in such presentations to avoid unnecessary concerns and or investigations.

\section{Acknowledgements}

The authors are grateful to the parents of the child who allowed to publish this case and those involved in the management.

\section{Disclosure Policy}

The author declare that there is no conflict of interest regarding the publication of this paper.

\section{References}

[1] Clementz GC, Mancini AJ. Nail matrix arrest following hand-foot-mouth disease: a report of five children. Pediatr Dermatol. 2000;17:7-11.

[2] Deeb M, Beach RA, Kim S. Onychomadesis following hand, foot, and mouth disease in a pregnant woman: A case report. SAGE Open Med Case Rep. 2019; 7:2050313X19845202. doi:10.1177/2050313X19845202

[3] Giordano L MC, de la Fuente L A, Lorca J MB, Kramer H D. Onychomadesis secondary to hand-foot-mouth disease: a frequent manifestation and cause of concern for parents. Rev
Chil Pediatr. 2018; $89 \quad$ (3):380-383. doi: 10.4067/S0370-41062018005000203. Spanish.

[4] Kuehnel NA, Thach S, Thomas DG. Onychomadesis as a Late Complication of Hand-Foot-Mouth Disease: A Case Series Shedding Light on Nail Shedding. Pediatr Emerg Care. 2017; 33 (11):e122-e123. doi: 10.1097/PEC.0000000000001292.

[5] Hand Foot and Mouth Disease (HFMD); Causes and transmission; Centre for Disease Control and Prevention; https://www.cdc.gov/hand-foot-mouth/about/transmission.htm 1; page reviewed Feb 2019.

[6] Shin YJi, Cho BK, and Park HJ. A Clinical Study of Nail Changes Occurring Secondary to Hand-Foot-Mouth Disease: Onychomadesis and Beau's Lines. Ann Dermatol. 2014; 26: 280-283. doi: 10.5021/ad.2014.26.2.280

[7] Yao X, Bian LL, Lu WW, Li JX, Mao QY, Wang YP, Gao F, Wu X, Ye Q, Li XL, Zhu FC, Liang Z. Epidemiological and etiological characteristics of herpangina and hand foot mouth diseases in Jiangsu, China, 2013-2014. Hum Vaccin Immunother. 2017; 13 (4):823-830.

[8] Corsino CB, Linklater DR. Herpangina. [Updated 2019 Apr 2]. In: StatPearls [Internet]. Treasure Island (FL): StatPearls Publishing; 2019 Jan-. Available from: https://www.ncbi.nlm.nih.gov/books/NBK507792/

[9] B'Krong NTTC, Minh NNQ, Qui PT, Chau TTH, Nghia HDT, Do LAH, Nhung NN, Van Vinh Chau N, Thwaites G, Van Tan L, van Doorn HR, Thanh TT. Enterovirus serotypes in patients with central nervous system and respiratory infections in Viet Nam 1997-2010. Virol. J. 2018;15 (1):69.

[10] Puenpa J, Mauleekoonphairoj J, Linsuwanon P, et al. Prevalence and characterization of enterovirus infections among pediatric patients with hand foot mouth disease, herpangina and influenza like illness in Thailand, 2012. PLoS One. 2014;9 (6):e98888. doi: 10.1371/journal.pone.0098888

[11] Boyarchuk, O., Volyanska, L., and Dyvonyak, O. Nail changes in case of enteroviral infections in western ukraine: a report of 34 cases. 2017; International Journal of Medicine and Medical Research, 2: 2413; https://doi.org/10.11603/ijmmr.

[12] Nguyen NT, Pham HV, Hoang CQ, et al. Epidemiological and clinical characteristics of children who died from hand, foot and mouth disease in Vietnam, 2011. BMC Infect Dis. 2014;18 (14):341. doi: 10.1186/1471-2334-14-341

[13] Chen YF, Hu L, Xu F, Liu CJ, Li J. A case report of a teenager with severe hand, foot, and mouth disease with brainstem encephalitis caused by enterovirus 71. 2019; BMC Pediatr. 9 (1):59. doi:10.1186/s12887-019-1428-4

[14] Beau JHS. Note sur certains caracteres de semeilogie retrospective presentes par les ongles. 1846. Arch Gen Med. 11:447-58.

[15] Venugopal SS, Murrell DF. Seasonal onychomadesis in an elderly gentleman. 2010. Dermatol Reports. 1 (1):e3. doi:10.4081/dr.2009.e3 